\title{
micromécanique des milieux granulaires approche expérimentale utilisant le modèle de Schneebeli
}

\author{
micromechanic of the granular media \\ experimental approach using the model of Schneebeli
}

\author{
GOURVES Roland \\ Maître de Conférences \\ MEZGHANI Fathi \\ Chercheur \\ Université Blaise Pascal de Clermont-Ferrand*
}

\section{Résumé}

La complexité des relations contrainte-déformation dans les milieux granulaires, qui est la conséquence de leur nature particulière, conduit à étudier leur comportement au niveau microstructural, c'est-à-dire à analyser les phénomènes discrets au niveau du grain ou d'un nombre restreint de grains.

Cette étude se situe dans ce contexte et a été menée sur un modèle bidimensionnel (petits rouleaux). Nous utilisons notamment une méthode expérimentale originale pour la mesure des forces de contact dans un tel milieu. L'étude de ces forces permet le calcul des contraintes.

Outre la caractérisation de la nature aléatoire de la contrainte au sein d'un milieu granulaire, cet article met en évidence l'influence de plusieurs paramètres sur les caractéristiques statistiques de cette dernière et permet une approche quantitative du passage entre le milieu discontinu et le milieu continu.

\section{Abstract}

Complexity of the stress-strain relationships of the granular media, which is the consequence of their discrete nature, leads to study their behaviour microstructurally or more precisely to analyse the discrete phenomenons considering one single particle or a restricted number of particles.

This work is placed in this context and is carried out upon a SCHNEEBELI'S bi-dimensional model. An original experiment method has been especially used for measuring the contact forces in such a medium. This method also makes possible the calculation of stresses.

In addition to the characterization of the random nature of the stress within the granular medium, this paper shows the influence of several parameters upon its statistical characteristics and permits a quantitative approach about the passage from non-continuous to continuous media.

\footnotetext{
- Groupe de Recherche Génie Civil, B.P. 45, 63170 Aubière.
} 


\section{INTRODUCTION}

Les travaux présentés dans cet article entrent dans le cadre de la mécanique des milieux granulaires et concernent plus particulièrement l'analyse statistique des contraintes macroscopiques au sein de tels matériaux.

En effet, en mécanique des sols les modèles de calcul s'appuient souvent sur les théories développées pour les milieux continus et permettent une prévision assez satisfaisante dans la plupart des cas. Toutefois, il est évident que les milieux granulaires formés de particules discernables à l'œil nu, voire même de dimensions importantes (sables, graviers, ....) ne peuvent répondre strictement aux hypothèses des milieux continus et il est donc nécessaire de leur appliquer une homogénéisation.

Nous tentons par le biais de ces études d'apporter une contribution à lamélioration des modélisations de ces milieux en tenant compte de leur nature discrète. Après avoir étudié les principales théories qui décrivent le comportement rhéologique des sols, nous nous sommes intéressés à l'analyse des phénomènes discrets au niveau du grain ou tout au moins d'un nombre relativement restreint de grains. Il s'agit en fait d'établir le lien entre les phénomènes discrets se produisant au niveau des particules et les propriétés rhéologiques du milieu considéré comme continu.

Les analyses effectuées sont essentiellement expérimentales et le matériau de SCHNEEBELI nous semble intéressant sur ce plan. II permet en effet d'avoir plusieurs renseignements tant au plan des déplacements qu'au plan des efforts. Dans ce dernier cas une technique de mesure des forces de contact a été mise au point à Clermont-Ferrand; elle est fondée sur l'étude du frottement longitudinal des rouleaux et permet de déterminer l'état de contrainte dans un tel milieu. Cette technique a été retenue pour notre étude.

\section{MODÉLISATION DES MILIEUX GRANULAIRES}

Le comportement mécanique des milieux granulaires a été le plus souvent schématisé tantôt comme élastique linéaire isotrope, tantôt comme parfaitement plastique avec critère de MOHR-COULOMB. Mais l'on s'est aperçu assez rapidement des limites de ces modèles qui ne correspondent qu'imparfaitement au comportement réel de ces milieux. Celui-ci n'est à aucun instant ni entièrement réversible, ni entièrement irréversible et répond bien plus à une loi continue de déformation associant à tout moment réversibilité et irréversibilité ; il est par conséquent beaucoup plus complexe.

Cependant, la mise en œuvre de puissantes méthodes numériques, en particulier la méthode des éléments finis, permet de prendre en compte des lois de comportement de plus en plus affinées. Dès lors, plusieurs recherches concernant la loi rhéologique à prendre en compte pour ces milieux granulaires ont été effectuées. Nous pouvons citer comme exemple les lois rhéologi- ques incrémentales telles qu'elles ont été décrites par DARVE à plusieurs reprises $(8,9,10,11)$.

Ces lois s'affranchissent de tout schéma de comportement et cherchent à suivre la nature pas à pas. La loi octolinéaire présente l'avantage de mieux décrire la non-linéarité du comportement des milieux granulaires et permet, grâce à l'hypothèse d'orthotropie, de prendre en compte une anisotropie généralement considérée comme suffisante pour un sol. Toutefois, ces modèles incrémentaux ne cernent pas la réalité; ils supposent que toutes les directions principales restent fixes et confondues. De plus, ils ne sont pas commodes à expliciter dès qu'il s'agit de préciser sur un trajet de chargement complexe les paramètres dont dépend la loi.

Néanmoins, dans toutes les modélisations utilisées, on considère les matériaux comme parfaitement continus. Il est évident que cette hypothèse ne correspond pas aux milieux granulaires formés de particules en contact les unes avec les autres et qui sont de formes, de dimensions et d'orientations diverses. La complexité des relations contraintes-déformations de ces milieux est en partie la conséquence de leur nature discrète dont il faut tenir compte.

\subsection{Description discrète des matériaux granulaires}

Il s'agit du développement d'une caractérisation statique qui consiste à définir le lien entre le tenseur des contraintes $\sigma$ et les forces de contact intergranulaires $\mathrm{F}^{k}$ (figure 1). Cette relation a été établie par WEBER (21) :

$$
\sigma=V^{-1} \Sigma 1^{k} \otimes F^{k}
$$

$\mathrm{V}$ étant le volume analysé supposé suffisamment grand.

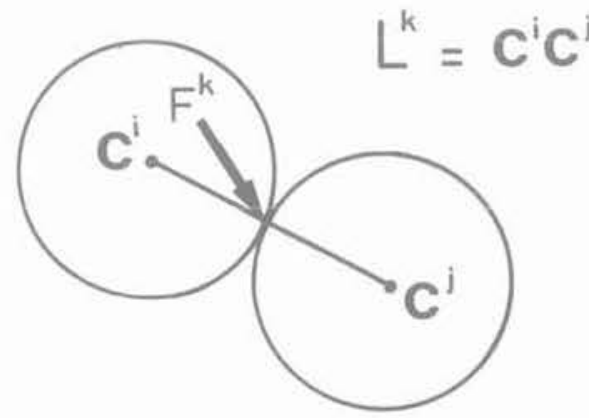

Fig. 1. - Contact et force intergranulaires.

Fig. 1. - Intergranular contact and force.

En partant de cette relation CAMBOU et Coll. $(5,6,7)$ ont défini un ensemble de variables internes $\lambda_{a \beta}$ susceptibles de représenter macroscopiquement l'état du matériau granulaire.

Pour un matériau bidimensionnel et dans une sollicitation à directions principales fixes, on obtient un état 
orthotrope caractérisé par $\lambda_{12}=\lambda_{21}=0$; deux variables $\lambda_{11}$ et $\lambda_{22}$ suffisent alors pour décrire l'état interne.

Ces variables d'état traduisent bien l'évolution de l'orientation des forces de contact dans le milieu (figure 2). Par ailleurs, les résultats de cette caractérisation statique montrent que l'évolution des variables $\lambda_{\text {if }}$ traduit l'adaptation de la structure de façon que le déviateur soit supporté pour une plus grande part par les composantes normales des forces de contact (figure 3).
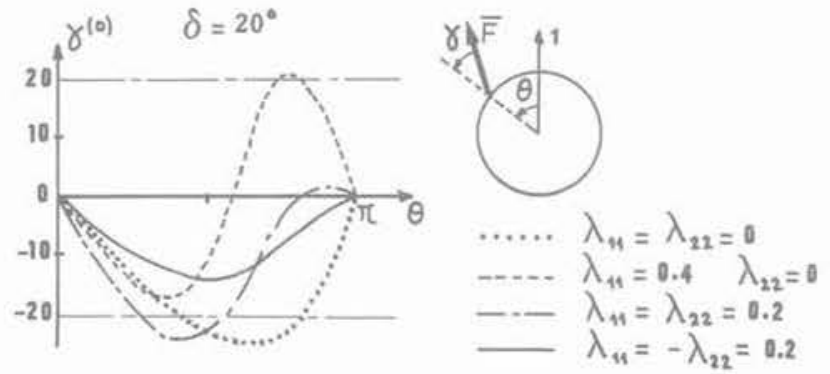

Fig. 2. - Distribution des orientations des valeurs moyennes des forces de contact (d'après Cambou).

Fig. 2. - Distribution of the orientations of the means values of the contact forces laccording to Camboul.

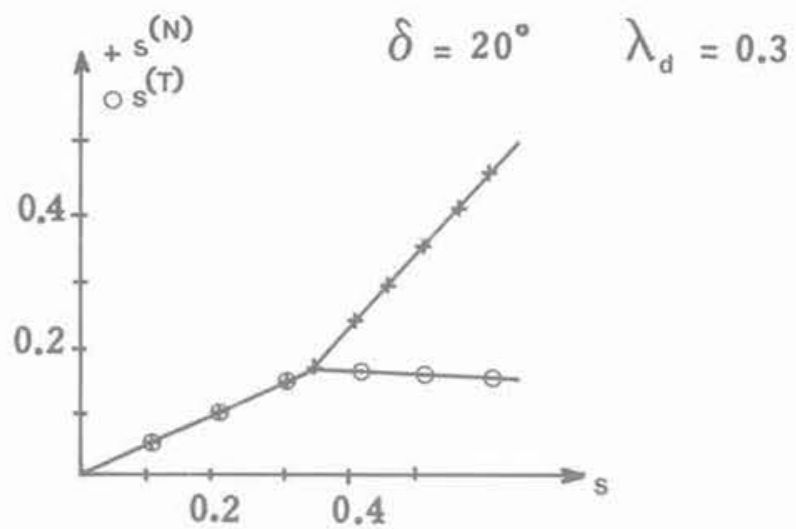

Fig. 3. - Contribution des composantes normales et tangentielles des forces de contact à l'angle de frottement mobilisés (d'après Cambou).

Fig. 3. - Contribution of the normals and tangentials components of contact forces to the mobilized friction angle $s$ laccording to Cambou)

\subsection{Modèle analogique de SCHNEEBELI}

Parallèlement à ces analyses théoriques du comportement mécanique des milieux granulaires, se développe une voie expérimentale utilisant le modèle analogique bidimensionnel de SCHNEEBELI. En effet, ce dernier a montré en 1956 la possibilité de réaliser un milieu pulvérulent, homogène, bidimensionnel et obéissant à la loi de COULOMB, par l'empilement de cylindres horizontaux, de plusieurs diamètres et de même longueur.

Un tel modèle permet l'étude de problème à deux dimensions et de nombreux auteurs $(3,18,19)$ l'ont utilisé notamment pour mieux comprendre les schémas de rupture du sol de fondation, l'équilibre limite des coins de poussée et de butée, etc. Il ne permettait que de visualiser les différents phénomènes et les résultats obtenus n'étaient donc que qualitatifs. Mais depuis quelques temps, cette technique connaît un renouveau avec lé développement des calculs par la méthode des éléments finis d'une part; le modèle n'est plus considéré comme un modèle réduit du problème étudié mais plutôt comme un objet particulier que l'on peut simuler par le calcul. Nous citerons dans ce cadre les travaux de TRATAPEL (20), BOULON et Coll. (4) et MONNET et Coll. (17). D'autre part, grâce à une technique originale de mesure des forces de contact $(12,13)$, il est possible d'obtenir l'état de contrainte en tous points du massif analogique. Nous verrons plus tard le principe de cette méthode.

Toutefois, les résultats obtenus tant qualitatifs que quantitatifs sont cependant limités par le danger qu'il y aurait d'extrapoler aux ouvrages réels en raison du non-respect des règles de similitude.

\subsection{Approche statistique}

Nous avons vu comment les méthodes numériques ont contribué à l'élaboration des modélisations des milieux granulaires mais il existe un autre moyen non moins important qui réside en l'utilisation des théories probabilistes et des méthodes statistiques.

En effet, un milieu granulaire présente des diversités de formes, de dimensions et d'orientations des contacts entre les grains. Il présente aussi des diversités de la minéralogie et donc de la loi de comportement propre à chaque particule. Tout ceci nous conduit alors à faire appel aux lois statistiques pour caractériser ce milieu.

C'est dans cette dernière catégorie que peuvent se situer les travaux présentés ici. Nous analysons plus particulièrement les phénomènes se rapportant à la notion de contrainte. Cette dernière, définie en un point dans les théories des milieux continus, doit se traduire par d'autres concepts dans un milieu granulaire.

\subsection{Contrainte macroscopique dans un milieu granulaire}

La contrainte macroscopique en un point d'un milieu granulaire est définie comme le rapport $\Delta \mathrm{F} / \Delta \mathrm{S}$, où $\Delta \mathrm{S}$ est une surface entourant le point et $\Delta \mathrm{F}$ la résultante de toutes les forces appliquées sur cette surface. La valeur de $\Delta S$ est finie et doit être suffisamment petite pour pouvoir l'assimiler à l'élément différentiel dans les traitements mathématiques, mais suffisamment grande de façon à ce que les discontinuités soient négligeables par rapport à sa taille. Ceci inclut donc l'idée d'homogénéisation locale du matériau. 
Considérons un matériau granulaire soumis à un système de forces extérieures : à celui-ci correspond, d'un point de vue déterministe, un état de contrainte équivalent qui est celui de la mécanique des milieux continus. Dans une approche probabiliste, la valeur de la contrainte macroscopique en un point ne sera pas unique mais sera une variable aléatoire dont la valeur moyenne $\sigma$ est égale à la contrainte du milieu continu équivalent.

\section{3. ÉTUDE À L'ÉCHELLE GLOBALE}

Avant de détailler les étapes ainsi que les résultats de l'analyse statistique effectuée nous exposons brièvement l'appareillage expérimental dont nous nous sommes servis ainsi que les résultats d'une étude portant sur le comportement mécanique global du milieu de SCHNEEBELI.

\subsection{Dispositif expérimental utilisé}

\subsubsection{Matériau de SCHNEEBELI}

Nous avons choisi d'utiliser des rouleaux en P.V.C. en raison du faible prix de revient, de leur légèreté et élasticité. Ces rouleaux sont de trois diamètres différents (2, 3 et $4 \mathrm{~mm}$ ) également répartis en poids, leur longueur est de $6 \mathrm{~cm}$. Le poids volumique du P.V.C. est de $1,3 \mathrm{daN} / \mathrm{dm}^{3}$, la densité moyenne du matériau en place évaluée par comptage sur photographie est de 1,1 , quant à l'angle de frottement interne mesuré à l'appareil biaxial, il est de l'ordre de $26^{\circ}$.

\subsubsection{Appareil de compression biaxiale}

Le schéma de principe de cet appareil est indiqué sur la figure 4. Il est constitué d'un moule carré, de $21,5 \mathrm{~cm}$ de côté, composé de quatre éléments rectifiés (mors) mobiles les uns par rapport aux autres. Deux cadres métalliques indépendants et articulés assurent la mise en charge du milieu selon deux directions perpendiculaires par un système vis-écrou. L'ensemble repose sur un plan lisse horizontal.

Les rouleaux sont disposés verticalement. Les efforts appliqués à l'aide du système de mise en charge se trouvent dans un plan horizontal et par conséquent le modèle est non pesant. Les efforts appliqués sont mesurés par deux anneaux dynamométriques; les déplacements respectifs des différentes parties du moule sont mesurés à l'aide de comparateurs.

L'abaissement d'une trappe située sous le moule permet d'éviter le frottement des rouleaux sur le fond et permet aussi leur déplacement longitudinal afin, comme nous le verrons plus loin, d'effectuer la mesure des contraintes.

Avant chaque essai, nous intercalons un lubrifiant à sec (téflon) entre les mors et les rouleaux de sorte qu'il n'y ait pas de frottement ou tout au moins qu'il soit négligeable. Nous évitons ainsi la création de voûtes de charge et les contraintes extérieures appliquées au milieu sont à tout instant de l'essai des contraintes principales.

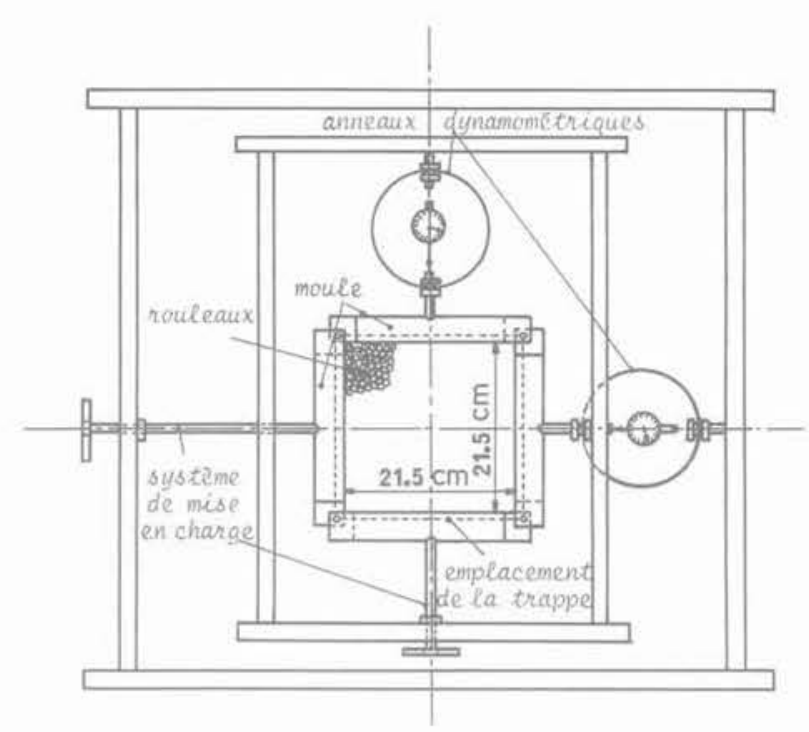

Fig. 4. - Appareil biaxial.

Fig. 4. - Biaxial apparatus.

\subsection{Comportement mécanique global du matériau de SCHNEEBELI}

Cette étude a été effectuée sur des chemins de contraintes monotones croissants à rapport de contraintes $R=\sigma_{1} / \sigma_{2}$ variable. Les essais suivent un chemin isotrope $\left(\sigma_{1}=\sigma_{2}\right)$ jusqu'à une certaine valeur, puis nous maintenons la contrainte dans la direction 2 constante tandis que la contrainte $\sigma_{1}$ continue à croître. Plusieurs valeurs de la contrainte latérale $\sigma_{2}$ ont été utilisées (figure 5).

Les mesures tirées de l'essai de compression biaxiale nous permettent la détermination des déformations $\varepsilon_{1}$ et $\varepsilon_{2}$ ainsi que le déviateur $\left(\sigma_{1}-\sigma_{2}\right) / 2$. Nous nous sommes aussi intéressés à l'étude des courbes reliant ces grandeurs physiques; ceci apparait sur la figure 6 .

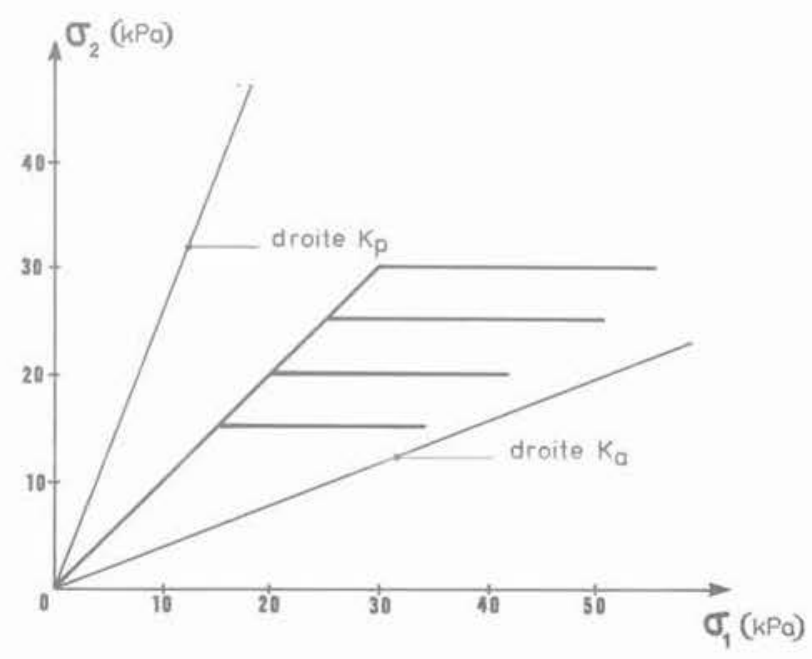

Fig. 5. - Chemins de contrainte étudiés. Fig. 5. - The studied stress-paths. 

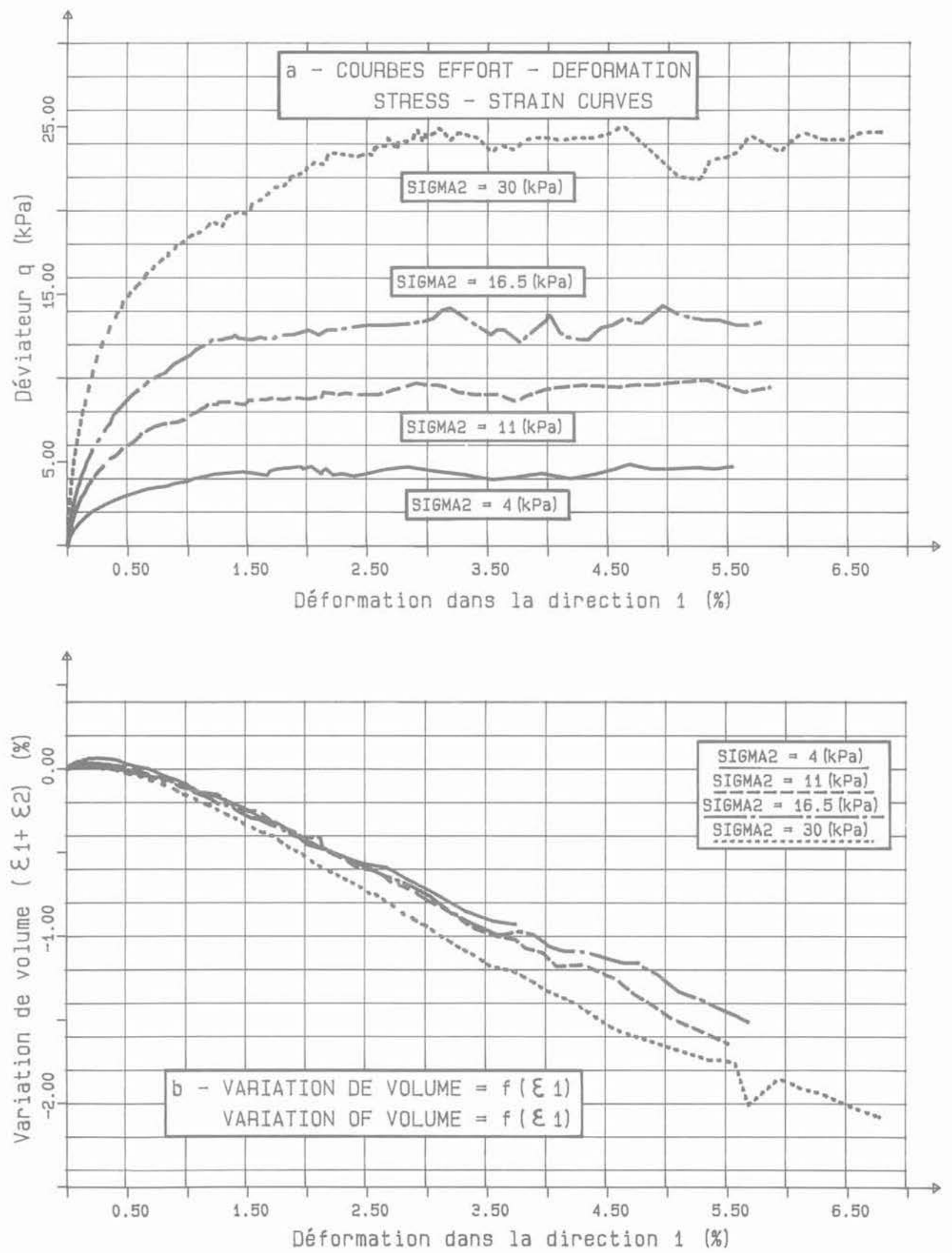


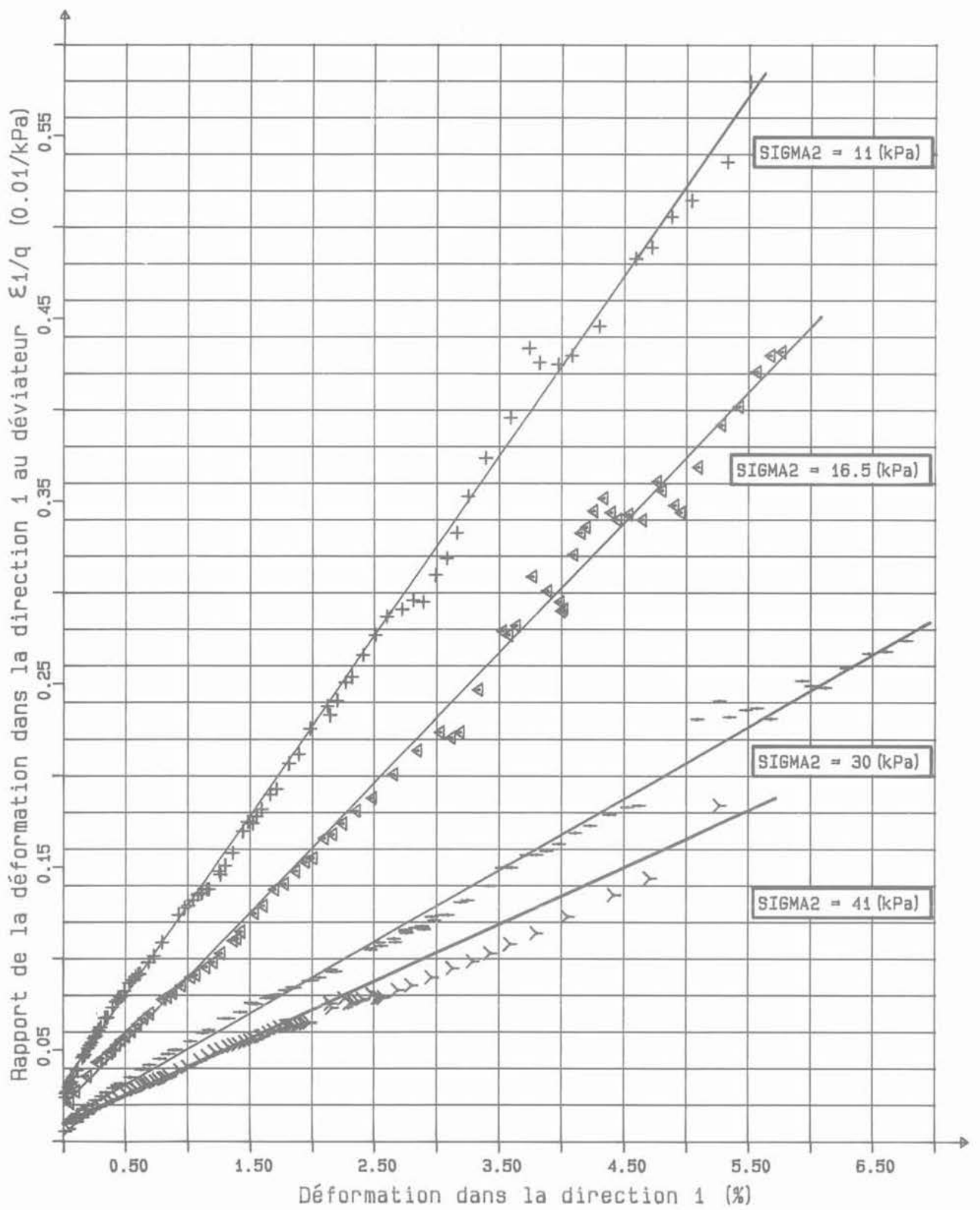

Fig. 7. - Lois hyperboliques - Transformations des courbes efforts - Déformation.

Fig. 7. - Hyperbolic laws - Stress -

Strain transformed curves. 
Les courbes effort-déformation de la figure 6a nous montrent la sensibilité de la résistance du milieu de SCHNEEBELI à la valeur de la contrainte latérale $\sigma_{2}$. Le matériau ne présente pas véritablement de pic de contrainte.

Les courbes de variation de volume de la figure $6 \mathrm{~b}$ mettent en évidence le phénomène de dilatance qui apparaît très nettement pour une valeur de la déformation latérale de l'ordre de $0,5 \%$.

A partir de ces courbes, il apparaît évident que le comportement du matériau de SCHNEEBELI lors d'un essai de compression biaxiale est analogue à celui d'un sable dense lors de l'essai de compression triaxiale conventionnelle.

Nous pouvons distinguer trois phases principales :

1. Phase de contraction $(0-0,5 \%)$ : réarrangement des rouleaux, compression du squelette, c'est le domaine de déformation homogène.

2. Phase de dilatance $(0,5-4 \%)$ : désenchevêtrement des particules, dilatance, pic en contrainte.

3. Phase d'état critique (au-delà de $4 \%$ ) : stabilisation des efforts, c'est le domaine d'écoulement plastique.

Par ailleurs, nous avons pu vérifier que pour le type de chemin de contraintes étudié, il est possible de décrire le comportement du milieu analogique par des formulations de type hyperbolique (figure 7).

\section{ANALYSE STATISTIQUE DES PHÉNOMÈNES DISCRETS}

Nous nous sommes limités à l'étude des problèmes se rapportant aux contraintes dans un milieu analogique en utilisant la méthode expérimentale due à FAUGE. RAS (12) et déjà évoquée (cf. § 2.3.).

\subsection{Principe de la méthode}

Le principe de cette méthode a déjà fait l'objet de plusieurs publications $(1,12,13,15)$, aussi nous ne rappelons ici que les principes essentiels. Cette méthode est fondée sur l'étude du frottement longitudinal des rouleaux constituant le massif. Elle consiste à mesurer la force nécessaire pour déplacer un ensemble de rouleaux selon leur axe longitudinal. Cette force est fonction du champ de contrainte au point de mesure $(12,13)$.

Dans le cas du biaxial et en adoptant les notations de la figure 8 , la relation entre la force mesurée et le champ de contrainte peut s'écrire :

$$
\begin{aligned}
& \mathrm{F}_{1}=\lambda \sigma_{1}+\mu \sigma_{2} \\
& \mathrm{~F}_{2}=\lambda \sigma_{2}+\mu \sigma_{1}
\end{aligned}
$$

Le fait que nous nous intéressons à un nombre important de rouleaux tient à ce que nous sommes en présence d'un milieu granulaire. Dans ce cas il est

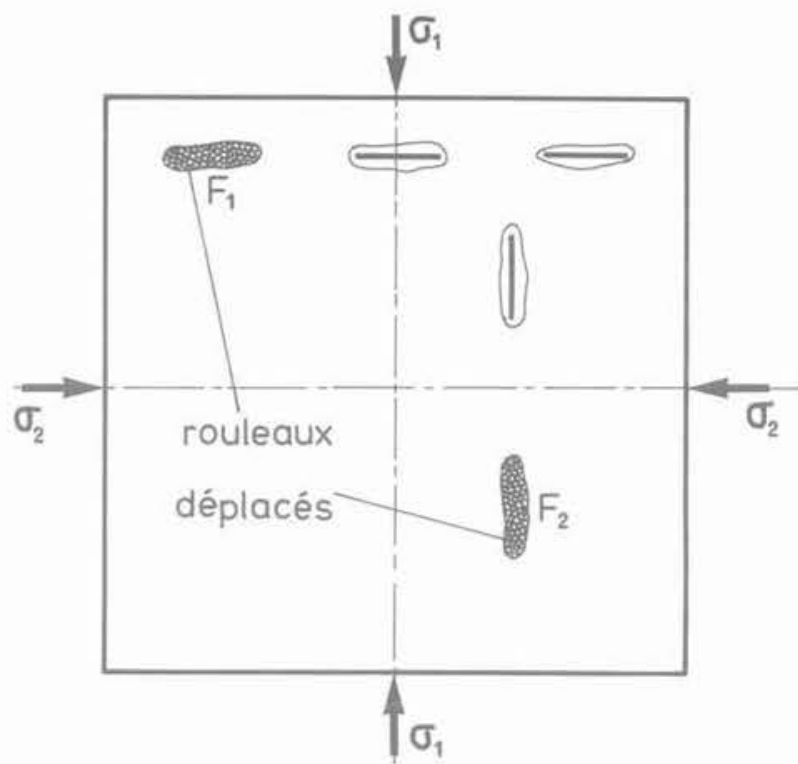

Fig. 8. - Notations et mesures des forces dans le biaxial.

Fig. 8. - Notations and measurement of forces in the biaxial.

nécessaire d'introduire la notion de contrainte macroscopique qui ne peut être utilisée qu'en considérant un élément assez représentatif du milieu.

Si nous appelons a découpe" la limite de l'empreinte créée dans le milieu par le déplacement d'un ensemble de cylindres, FAUGERAS (12) a mis en évidence les avantages de l'utilisation d'une découpe obtenue par l'intermédiaire d'une plaque d'épaisseur très faible par rapport à sa largeur.

Dans la relation (2) $\mathrm{F}_{1}$ et $\mathrm{F}_{2}$ sont mesurées à l'aide d'un capteur de force. $\lambda$ et $\mu$ sont deux coefficients, homogènes à une surface, qui dépendent des caractéristiques géométriques de l'empreinte et de la nature des matériaux. Des travaux plus récents (16) montrent que $\lambda$ et $\mu$ dépendent aussi de la géométrie de l'assemblage.

\subsection{Champ de contrainte macroscopique}

Il est inutile de rappeler que pour cette étude nous avons été amenés à abandonner le modèle déterministe pour une formulation probabiliste faisant intervenir les incertitudes affectant les matériaux réels. L'étude a été effectuée dans les deux domaines avant et après dilatance mis en évidence lors de l'étude à l'échelle globale.

\subsubsection{Mise en cuvre des essais}

Les essais au biaxial se déroulent de la façon suivante : - application d'un champ de contraintes théoriques constant $\left(\sigma_{1}, \sigma_{2}\right)$;

- mesure des forces $F_{1}$ en $n$ points différents (figure 8); 
- déchargement du système et réarrangement des rouleaux;

- application du même champ de contraintes théoriques $\left(\sigma_{1}, \sigma_{2}\right)$;

- mesure des forces $F_{2}$ en $n$ points différents;

- nouveau déchargement et régénération du milieu.

Ces opérations sont répétées un certain nombre de fois afin d'avoir un nombre de mesures permettant une exploitation statistique convenable. Nous avons suivi le même type de chemin de contraintes que lors de l'étude du comportement global. Les contraintes macroscopiques se déterminent à partir de ces mesures à l'aide des relations suivantes :

$$
\begin{aligned}
& \sigma_{1}=\left(\lambda F_{1}-\mu F_{2}\right)\left(\lambda^{2}-\mu^{2}\right)^{-1} \\
& \sigma_{2}=\left(\lambda F_{2}-\mu F_{1}\right)\left(\lambda^{2}-\mu^{2}\right)^{-1}
\end{aligned}
$$

\subsubsection{Influence du nombre de mesures}

Le nombre de mesures nécessaires pour obtenir un point expérimental dans l'espace des contraintes principales $\left(\sigma_{1}, \sigma_{2}\right)$ est un facteur très important dans le contexte qui nous intéresse, dans la mesure où tous les résultats que nous obtiendrons par la suite découleront d'une analyse statistique. ASTIER (1) et LEBROU (15) ont montré que des échantillons comportant une cinquantaine d'éléments permettent d'obtenir un ensemble de valeurs statistiquement fiable (figure 9).

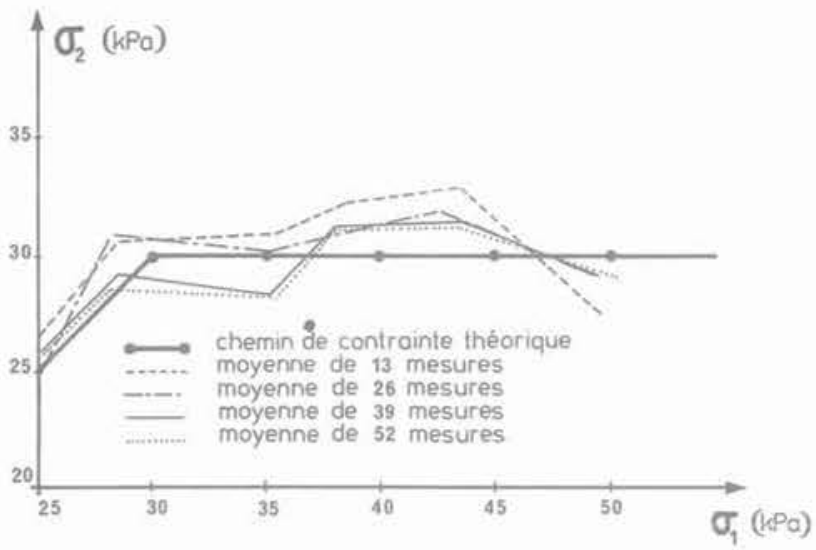

Fig. 9. - Influence du nombre de mesures sur

la validité du chemin de contrainte expérimental ( $d$ 'après Astier).

Fig. 9. - Influence of the measurements number upon the validity of the experimental stress-path laccording to Astier)

\subsubsection{Influence de la position du point de mesure}

Dans toutes nos expérimentations le biaxial se trouve soumis à un champ de contrainte globalement homogène. Dans une approche probabiliste cela se traduit par une même probabilité sur la valeur du champ de contraintes macroscopiques pour tous les points situés à l'intérieur du milieu.
D'autres travaux $(1,2,15)$ montrent que l'on peut admettre que les contraintes mesurées sont indépendantes du point de mesure dans le biaxial (figure 10) Les variations qu'on peut enregistrer d'un point à lautre sont en fait dues au milieu lui-même et à sa nature granulaire.
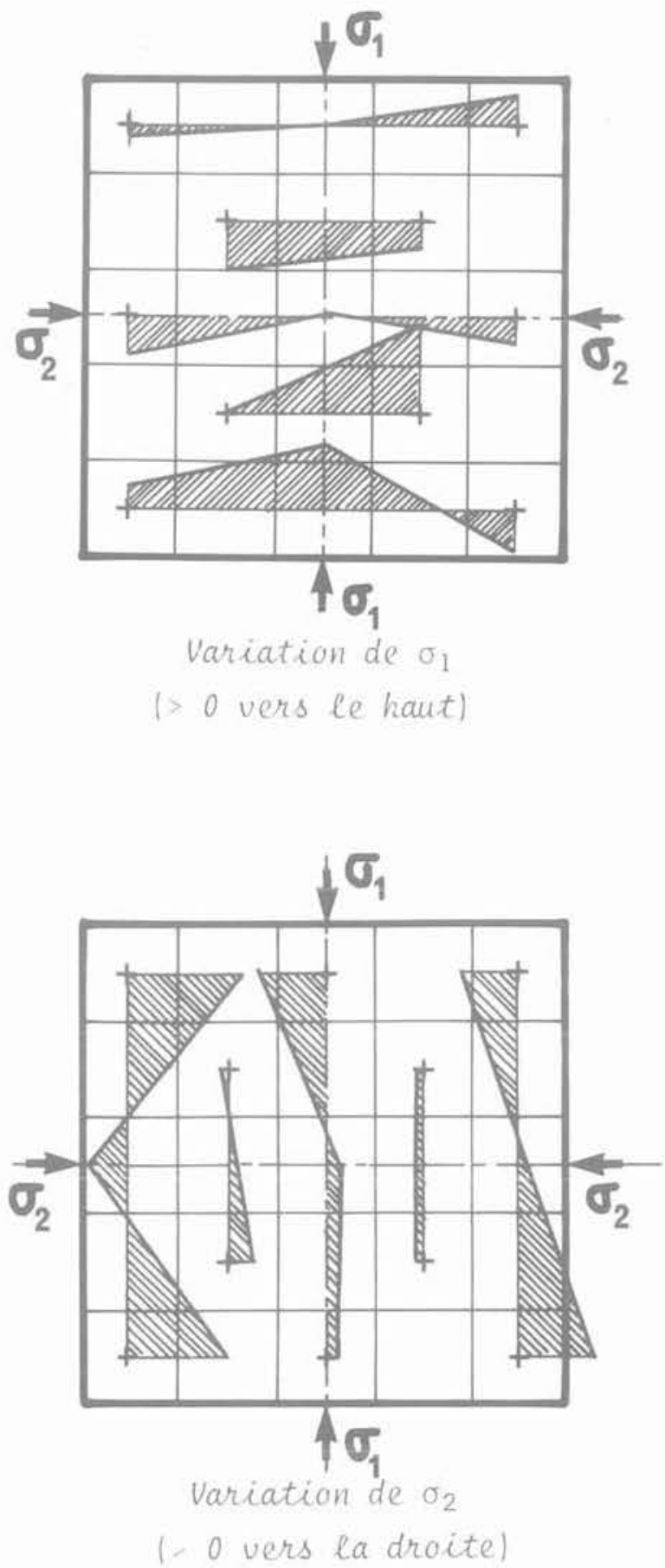

Fig. 10. - Variation de $\sigma_{1}$ et $\sigma_{2}$ dans le biaxial (d'apres Astier et Coll.).

Fig. 10. - Variation of $\sigma_{1}$ and $\sigma_{2}$ in the biaxial (according to Astier and al.). 


\subsubsection{Aspect aléatoire de la contrainte macroscopique}

La figure 11 présente un exemple type des résultats tirés à partir de nos mesures à l'aide d'une plaque de $2 \mathrm{~cm}$ de large. Sur ces graphiques nous avons porté dans le plan (p, q) la comparaison entre le chemin des contraintes théoriques et celui des contraintes moyennes expérimentales. Celle-ci fait apparaître une différence relativement faible entre les deux courbes. Mais cette comparaison ne porte que sur une valeur caractéristique des échantillons de mesures qui eux présentent, comme on peut s'y attendre et comme l'indique la figure 12 , un nuage autour de cette valeur moyenne ne présentant aucune corrélation particulière entre les deux variables statistiques $\mathrm{p}$ et $\mathrm{q}$.

Il importe de souligner qu'avec notre technique expérimentale nous pouvons déterminer la valeur du déviateur $q$, bien que nous ne fassions intervenir que les composantes normales des forces de contact. Ce résultat vient confirmer les résultats des analyses théoriques de CAMBOU (7) qui montrent que le déviateur est supporté pour une plus grande part par les composantes normales des forces de contact.

Les deux résultats exposés ci-dessus traduisent bien le caractère aléatoire de la contrainte macroscopique au sein du milieu analogique. Celle-ci est définie comme étant égale à la somme des composantes normales des forces s'exerçant sur les particules divisée par l'aire de la facette macroscopique considérée. Dans notre cas, la taille de la plaque utilisée correspond à celle de la facette macroscopique. Il est donc évident que la dispersion des mesures est d'autant plus importante que la taille de la facette macroscopique prise en compte est petite par rapport à la taille moyenne des grains. Ceci a été confirmé lors des études de MEZGHANI (16) dont nous présentons quelques exemples de résultats ci-après.

\subsubsection{Passage du milieu discontinu au milieu continu}

Le passage du milieu discontinu au milieu continu peut se traduire par le rapport de la taille moyenne des grains à la taille des plaques. En effet plus la taille de la plaque est petite moins la facette est représentative du milieu donc plus on tend vers le milieu discontinu alors que dans le cas contraire on aura tendance à aller vers le milieu continu.

Ceci apparaît nettement sur les histogrammes de la figure 13 , ces derniers ne font que confirmer toutes les considérations précédentes. Plus la taille de la plaque est grande, plus la distribution est serrée et les histogrammes de fréquence en nombre des échantillons des valeurs obtenues se réduisent à quelques intervalles et admettent un maximum correspondant à la valeur moyenne. Pour les plaques de petite taille la distribution a tendance à s'étaler et nous avons des maximums beaucoup moins marqués. Toutefois, il est à remarquer que les histogrammes obtenus sont toujours compris dans des bornes bien nettes quelle que soit la taille de la plaque. FAUGERAS et GOURVES (14) ont effectué des essais au niveau des grains et ont alors proposé dans ce cas une loi de répartition uniforme des forces intergranulaires. Nous pouvons effectivement

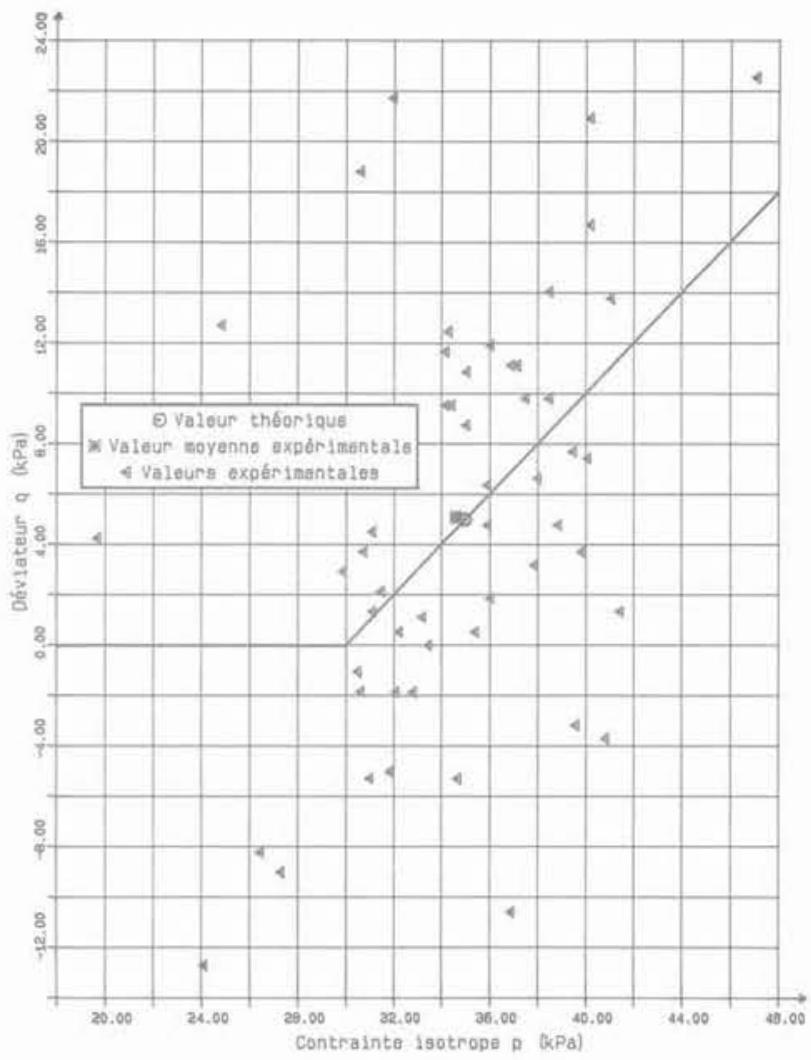

Fig. 11. - Chemins de contrainte en p. q-

Résultats obtenus á la plaque de $2 \mathrm{~cm}$.

Fig. 11. - Stress-paths in p. q-Results obtained with the $2 \mathrm{~cm}$ plate.

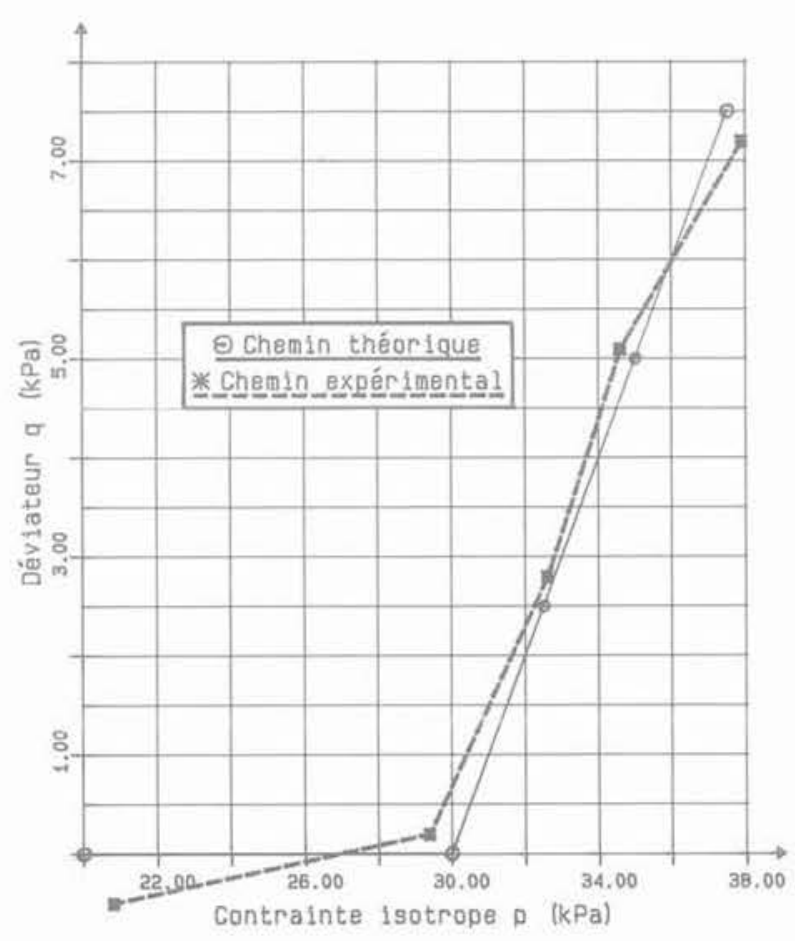

Fig. 12. - Diagramme de dispersion - Plaque de $2 \mathrm{~cm}$.

Fig. 12. - The dispersion diagram - A $2 \mathrm{~cm}$ plate. 


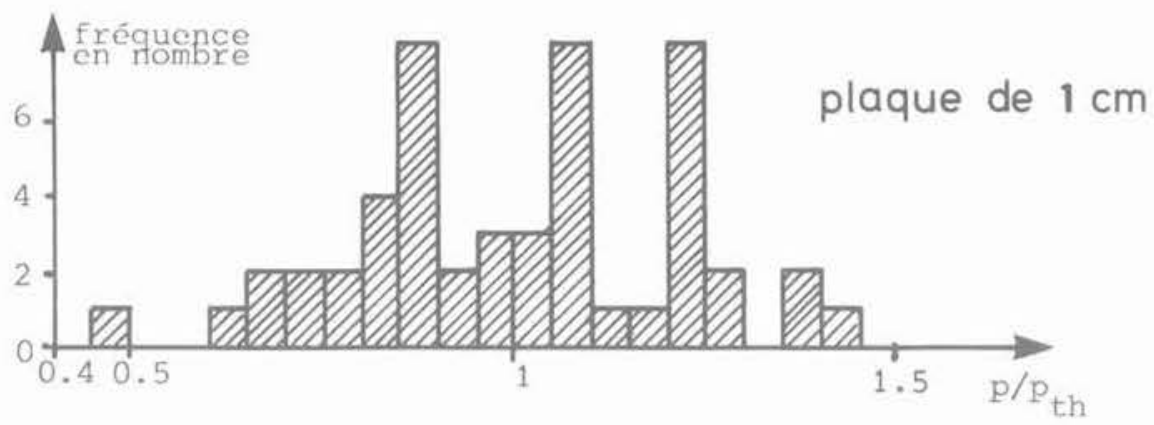

plaque de $2 \mathrm{~cm}$
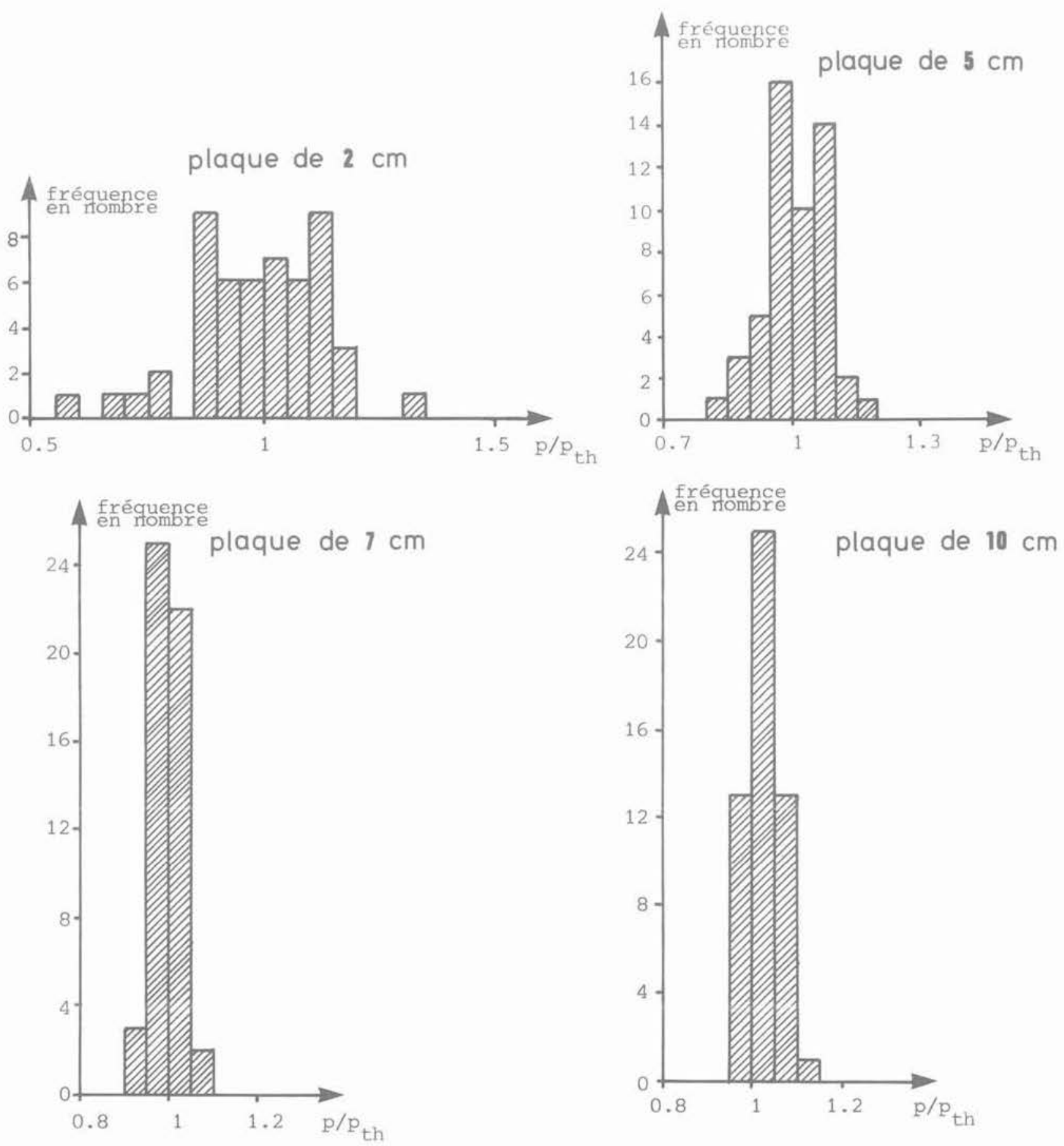

Fig. 13. - Histogrammes des échantillons de valeurs relatifs à l'état de contrainte

Fig. 13. - Histograms of the value samples related $\sigma_{1}=40 \mathrm{kPa}, \sigma_{2}=30 \mathrm{kPa}$. to the stress state $\sigma_{1}=40 \mathrm{kPa}, \sigma_{2}=30 \mathrm{kPa}$. 
supposer qu'une telle loi est acceptable. Puisqu'il ne peut $y$ avoir de forces de traction entre les grains, la conséquence en serait que la force intergranulaire est limitée au double de la valeur moyenne. Cette considération conduit alors à dire que les effets de concentration de contraintes seraient nécessairement limités.

Le passage du milieu discontinu au milieu continu se traduit aussi très bien par le graphique de la figure 14 quil nous donne en quelque sorte une certaine mesure de ce passage. En effet, le milieu continu a par définition un coefficient de variation nul (à une erreur expérimentale près). Nous pouvons donc admettre que le matériau granulaire est assimilable à un milieu continu tant que ce coefficient est faible. Il est d'usage courant d'admettre que tel est le cas dès qu'on s'intéresse à une dizaine de particules ce qui correspond dans notre cas à la plaque de $2 \mathrm{~cm}$.

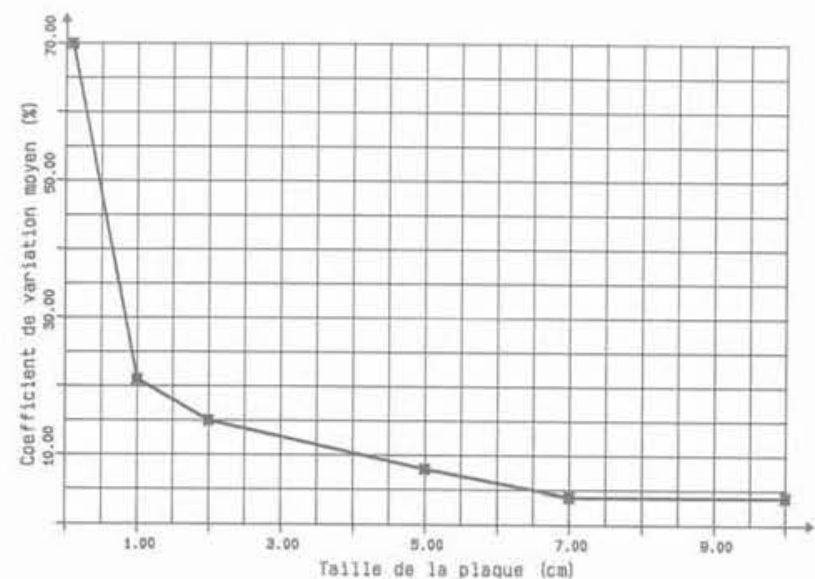

Fig. 14. - Coefficient de variation moyen de la contrainte isotrope $p$ en fonction de la taille de la plaque.

Fig. 14. - The mean coefficient of variation of the isotropic stress $p$ in terms of the plate size.

\subsubsection{Influence de la dilatance}

L'étude dans le domaine après dilatance a été beaucoup moins approfondie que dans le domaine des déformations homogènes pour une raison purement technique liée à l'appareil de compression biaxiale luimême. En effet, ce dernier risque d'être une source d'erreur non négligeable dans ce domaine et il faudrait lui faire subir quelques modifications pour que l'on puisse étudier correctement l'influence de la dilatance.

Toutefois, les premiers résultats obtenus montrent que les coefficients $\lambda$ et $\mu$, qui gardent des valeurs constantes avant le seuil de dilatance, présentent, dès que ce seuil est dépassé, une évolution pouvant être due à une variation de l'état interne. De plus, nous aurions pu penser que l'atteinte du seuil de dilatance provoquerait une redistribution des efforts. Les résultats obtenus (16) ne nous permettent pas de conclure en ce sens et tendraient au contraire à prouver que les caractéristiques de dispersion sont les mêmes avant et après dilatance.

\section{CONCLUSION}

L'approche présentée ici montre bien la nécessité de l'analyse au niveau de la particule. Le modèle de SCHNEEBELI est à notre sens un outil très intéressant sur ce plan.

En effet ce dernier, dont le caractère analogique a été vérifié lors de notre étude à l'échelle globale, présente l'avantage d'être aisé à mettre en ceuvre et permet donc d'effectuer des analyses statistiques dont l'intérêt ne cesse d'être prouvé.

Cette étude nous permet d'affirmer le caractère aléatoire de la contrainte au sein d'un milieu granulaire. Celle-ci est une variable aléatoire qui suit donc une loi de distribution statistique donnée pouvant être, dans un premier temps, approchée par deux paramètres :

- une caractéristique de position (moyenne o) égale à la contrainte dans le milieu continu équivalent;

- une caractéristique de dispersion (écart-type) fonction de la taille de la facette macroscopique prise en compte ainsi que de la nature du milieu.

Par ailleurs, si la nature aléatoire des milieux granulaires a été bien caractérisée, d'autres points le sont beaucoup moins. Il conviendrait d'analyser notamment le phénomène de dilatance et l'évolution d'état qui en découle, ainsi que la concentration de contrainte ne pouvant se produire de la même façon que dans les milieux continus qui admettent une certaine résistance à la traction.

\section{BIBLIOGRAPHIE}

1. ASTIER R. (1983), Milieux granulaires : approche probabiliste des états de contraintes et de déformations sur modèle analogique. Thèse présentée à l'Université de Clermont II, soutenue le 14 décembre 1983, pour obtenir le grade de Docteur-Ingénieur.

2. ASTIER R., FAUGERAS J.C., GOURVES R. (1984), A statistical analysis of the states of stress within a bidimensional model. Octobre 1984, Advances in Rheology. 1. Theory. Edited by B. MENA, A. GARCIA-REJON, C. RANGELNAFAILE. Universidad Nacional Autonoma de Mexico.

3. BIAREZ J. (1962), Contribution à l'étude des pro. priétés mécaniques des sols et des matériaux pulvérulents. Thèse présentée à la Faculté des Sciences de l'Université de Grenoble, soutenue en 1962 pour obtenir le titre de Docteur ès Sciences. 
4. BOULON M. et al. (1977), Comportement d'un écran et d'un pieu - essai, calculs. 1977, IX ${ }^{e}$ Congrès International de Mécanique des Sols et des Travaux de Fondations. Tokyo, vol. 1.

5. CAMBOU B. (1984), Mécanique des matériaux granulaires, lapproche microstructurale. Décembre 1984. Cours de l'École d'Hiver des Géomatériaux, C.N.R.S., I.M.G., Aussois.

6. CAMBOU B., SIDOROFF F. (1985), Description de létat d'un matériau granulaire par variables internes statiques à partir d'une approche discrète. Journal de Mécanique Théorique et Appliquée, 1985.

7. CAMBOU B. (1985), Les micromécanismes de déformations plastiques dans les sols granulaires. Revue Française de Géotechnique, $n^{\circ} 31$, $2^{e}$ trimestre 1985, pp. 39-51.

8. DARVE F. (1974), Contribution à la détermination de la loi rhéologique incrémentale des sols. Thèse présentée à l'Université Scientifique et Médicale de Grenoble, soutenue le 24 juin 1974 pour obtenir le grade de Docteur-Ingénieur.

9. DARVE F., LABANIEH S. (1980), Comporte. ment mécanique des milieux granulaires en liaison avec leur structure. Décembre 1980, Comportements Rhéologiques et Structures des Matériaux, XVe Colloque Annuel du Groupe Français de Rhéologie, Paris.

10. DARVE F., LABANIEH S. (1982), Incremental constitutive law for sands and clays : simulations of monotonic and cyclic tests. International Journal for Numerical and Analytical Methods in Geomechanics, vol. 6, 1982, pp. 243-275.

11. DARVE F., LABANIEH S. (1982), An incremental non-linear constitutive law and cyclic behaviour of sands. Septembre 1982, International Symposium on Numerical Models in Geomechanics, Zurich.

12. FAUGERAS J.C. (1979), L'essai de compressibilité des sols au pénétromètre statique et son inter. prétation sur modèle analogique. Thèse présentée à l'Université Paul Sabatier de Toulouse, soutenue le 25 septembre 1979 pour obtenir le grade de Docteur de $3^{\mathrm{e}}$ cycle.

13. FAUGERAS J.C., GOURVES R. (1980), Mesure des contraintes au sein d'un massif analogique de SCHNEEBELI. Revue Française de Géotechnique, $\mathrm{n}^{\circ} 11$, mai 1980 , pp. 5-16.
14. FAUGERAS J.C., GOURVES R. (1980), Distribution des efforts sur les particules d'un milieu granulaire soumis à un champ isotrope. Décembre 1980, Comportements Rhéologiques et Structures des Matériaux, Groupe Français de Rhéologie, $\mathrm{XVe}$ Colloque Annuel, Paris.

15. LEBROU Ph. (1983), Contribution à l'étude des champs de contraintes et de déformations au sein d'un milieu granulaire. Mémoire présenté au Centre Universitaire des Sciences et Techniques de Clermont II, soutenue en juin 1983 pour obtenir le diplôme d'ingénieur.

16. MEZGHANI F. (1987), Milieux granulaires -analyse statistique de l'état de contrainte macroscopique au sein d'un milieu analogique. Thèse présentée à l'Université de Clermont II, soutenue le 5 février 1987 pour obtenir le titre de Docteur de l'Université de Clermont II.

17. MONNET J., KASTNER R., COULET C. (1980), Détermination d'une loi de comportement contrainte-déformation du matériau de SCHNEE. BELI. Octobre 1980, Mécanique des Sols Appliquées, Communications présentées aux Journées Universitaires « Génie Civil » à l'Institut National des Sciences Appliquées de Lyon.

18. NHIEM T.V. (1971), Observations cinématiques et statiques du phénomène de soulèvement oblique des fondations d'ancrage en milieu pulvérulent bidimensionnel. Bulletin de liaison des Laboratoires des Ponts et Chaussées, numéro spécial * Le comportement des sols avant la rupture ", mai 1971 , pp. 127-137.

19. ORTHOLARY M. (1979), Étude du schéma de rupture sous une fondation superficielle rigide. Mémoire présenté au Centre Universitaire des Sciences et Techniques de Clermont II, soutenu en juin 1979 pour obtenir le diplôme d'ingénieur.

20. TRATAPEL G. (1977), Contribution à l'étude du comportement mécanique d'un milieu analogique bidimensionnel. Thèse présentée à l'Université Scientifique et Médicale et l'Institut National Polytechnique de Grenoble, soutenue le 18 juillet 1977 pour obtenir le grade de Docteur-Ingénieur.

21. WEBER J. (1966), Recherches concernant les contraintes intergranulaires dans les milieux pulvérulents. Bulletin de liaison des Laboratoires des Ponts et Chaussées, n²0, juillet-août 1966 , pp. 3.1-3.20. 